\title{
Original
}

\section{Papilomas invertidos nasosinusales. Revisión de 10 años}

\section{Jhonder Xavier Salazar Guilarte*, Juan Ramón Paredes Osado, José Mauri Barberá y Juan Ramón Gras Albert}

Servicio de Otorrinolaringología, Hospital General Universitario de Alicante, Alicante, España

\section{INFORMACIÓN DEL ARTÍCULO}

Historia del artículo:

Recibido el 4 de agosto de 2011

Aceptado el 21 de septiembre de

2011

On-line el 28 de octubre de 2011

\section{Palabras clave:}

Papiloma invertido nasosinusal

Tumor benigno nasosinusal

Cirugía endoscópica nasal

Keywords:

Sinonasal inverted papilloma

Sinonasal benign tumor

Endoscopic surgery

\section{R E S U M E N}

Objetivos: Analizar nuestra experiencia en el diagnostico y tratamiento del papiloma invertido (PI), especialmente en relación a recientes cambios y estrategias en los abordajes quirúrgicos.

Material y método: Se realizó un estudio retrospectivo durante 10 años en pacientes diagnosticados de PI consecutivamente seleccionados. El tiempo medio de seguimiento fue de 36 meses.

Resultados: 15 casos diagnosticados por estudio anatomopatológico predominantemente hombres, y en estadio T2 según la clasificación Krouse. La localización primaria más común fue el seno etmoidal y maxilar. La tasa de recidiva del total de pacientes fue de $40 \%$, con predominio de los pacientes sometidos a abordaje mixto (abierto y endoscópico).

Conclusiones: La modalidad de tratamiento depende de la localización y extensión de la enfermedad. Se recomienda un seguimiento anual endoscópico para valorar los resultados del tratamiento y, de por vida debido a posibles recurrencias tardías.

(c) 2011 SECOM. Publicado por Elsevier España, S.L. Todos los derechos reservados.

\section{Sinunasal inverted papilloma. A 10 year review}

\section{A B S T R A C T}

Aim: To analyze our experience in the diagnostic and treatment of inverted papilloma (IP), especially based on recent changes and strategies through surgical approach.

Material and method: This is a retrospective chart study over a 10-year period in patients with IP consecutively selected. The mean follow-up period was 36 month.

Results: 15 cases diagnosed by anatomopathological study predominantly males, and T2 stage according to Krouseřs classification system. Most common primary localization of the tumor was ethmoid and maxillary sinus. The recurrence rate was $40 \%$, predominantly in patients with combined approach (external and endoscopic).

Conclusions: The treatment modality is related to the location and extent of the disease. Annual endoscopic follow-up observation is recommended for the preliminary report on the treatment results of this condition and, lifelong follow-up for possible late recurrences. ๑) 2011 SECOM. Published by Elsevier España, S.L. All rights reserved.

* Autor para correspondencia.

Correo electrónico: jhoxasagui@hotmail.com (J.X. Salazar Guilarte).

1130-0558/\$ - see front matter @ 2011 SECOM. Publicado por Elsevier España, S.L. Todos los derechos reservados.

doi:10.1016/j.maxilo.2011.09.004 


\section{Introducción}

A diferencia de los papilomas evertidos y los oncocíticos, el PI nasosinusal representa el tipo relativamente más frecuente de estas tumoraciones ${ }^{1-4}$, siendo la pared lateral nasal su lugar predilecto de crecimiento $^{1,2}$. A pesar de estar presente en la sospecha clínica diaria de cualquier otorrinolaringólogo, supone apenas un 0,5-7\% de todas las tumoraciones nasales y afecta principalmente a varones, entre la $5^{\text {ta }}$ y $6^{\text {ta }}$ década de la vida ${ }^{1,4,5}$.

Hoy día se sabe que no se trata realmente de un papiloma, sino de una degeneración metaplásica severa de la mucosa que crece hacia el estroma subyacente y que resulta difícil de diferenciar de un carcinoma escamoso de bajo grado ${ }^{2}$, por lo que clásicamente ha sido categorizado en tres tipos, PI benigno, PI con displasia y/o carcinoma in situ, y PI con carcinoma de células escamosas ${ }^{7}$. Su etiología es incierta, aunque se ha publicado ${ }^{2,5-7}$ que cuando se relaciona en fases iniciales con el virus del papiloma humano (VPH) 16 y 18 su incidencia (entre 0 y 70\%) de transformación maligna a carcinoma es mayor.

Recientemente se ha señalado que la desmogleina 3 que está presente en $90 \%$ de los carcinomas escamoso celulares, también se localiza en ciertas áreas del PI (53\%) por lo que su hallazgo puede servir como predictor de transformación maligna ${ }^{7}$.

Varios sistemas de estadiaje y categorización se han propuesto ${ }^{2,3}$, pero hasta ahora ninguno unificado que determine la severidad de la enfermedad, siendo el más usado el de Krouse ${ }^{12}$.

Tanto la tomografía computarizada (TC) como la resonancia magnética (RM) nos permiten delimitar la extensión de la lesión antes de la cirugía, distinguiéndola del tejido inflamatorio circundante bien mediante la captación de contraste y la detección del patrón columnar que nos muestra la RM potenciada en T2 o a través de la osteolisis radiológica que se aprecia en la $\mathrm{TC}^{2,6}$. Sin embargo, esta última no es suficiente para asegurar el diagnóstico de PI, porque puede estar presente hasta en $53 \%$ de pacientes con sinusitis crónica ${ }^{6}$.

\section{Materiales y métodos}

Se realizó un estudio retrospectivo con la revisión de historias clínicas desde 1998 hasta 2008. A través de un protocolo se recogen datos clínico patológicos como edad, sexo, localización, tratamiento realizado y evolución postquirúrgica. Las lesiones se estadificaron según el sistema propuesto por Krouse ${ }^{12}$. Se presenta el análisis descriptivo de los resultados.

\section{Resultados}

En el período de estudio fueron tratados en nuestro hospital 15 papilomas invertidos, de los cuales 11 eran hombres (73,3\%) y 4 mujeres $(26,7 \%)$, con una edad media de 54 años (rango: 30-74 años). El síntoma principal fue insuficiencia respiratoria nasal unilateral $(86,7 \%)$ seguido de algias faciales (cefalea) y epistaxis con $40 \%$ y $26,7 \%$ respectivamente. El $60 \%$ de los pacientes presentaba papilomas en la fosa nasal izquierda. Endoscópicamente 66,6\% de las lesiones presentaban un aspecto pólipo/vegetante grisáceo-pálido (fig. 1 B).

Las tumoraciones se extendían comprometiendo diferentes localizaciones; etmoides (anterior y posterior) con $86,7 \%$, seguida de seno maxilar (60\%) y esfenoides (33,3\%). El tiempo medio desde la presentación de los síntomas hasta el diagnóstico y tratamiento fue de 59 meses. En los 15 pacientes analizados se realizó TC y en 6 (40\%) se complementó con RM.

Según la clasificación de Krouse, 7 (46,7\%) pacientes se encontraban al momento del diagnóstico en estadio I, 4 (26,7\%) en estadio II, 3 (20\%) en estadio III y 1 (6,6\%) en estadio IV.

Once pacientes fueron operados por abordaje endoscópico, 1 únicamente por etmoidectomía externa y 3 se sometieron en diferentes oportunidades a ambos procedimientos.

Todos los pacientes firmaron un consentimiento informado sobre el procedimiento a realizárseles y se les avisó de la posibilidad de transformación maligna, recurrencia y prolongados seguimientos evolutivos.

La tasa de recidiva total fue del $40 \%$, observada en $4(26,6 \%)$ pacientes del grupo que se sometieron a abordaje externo y

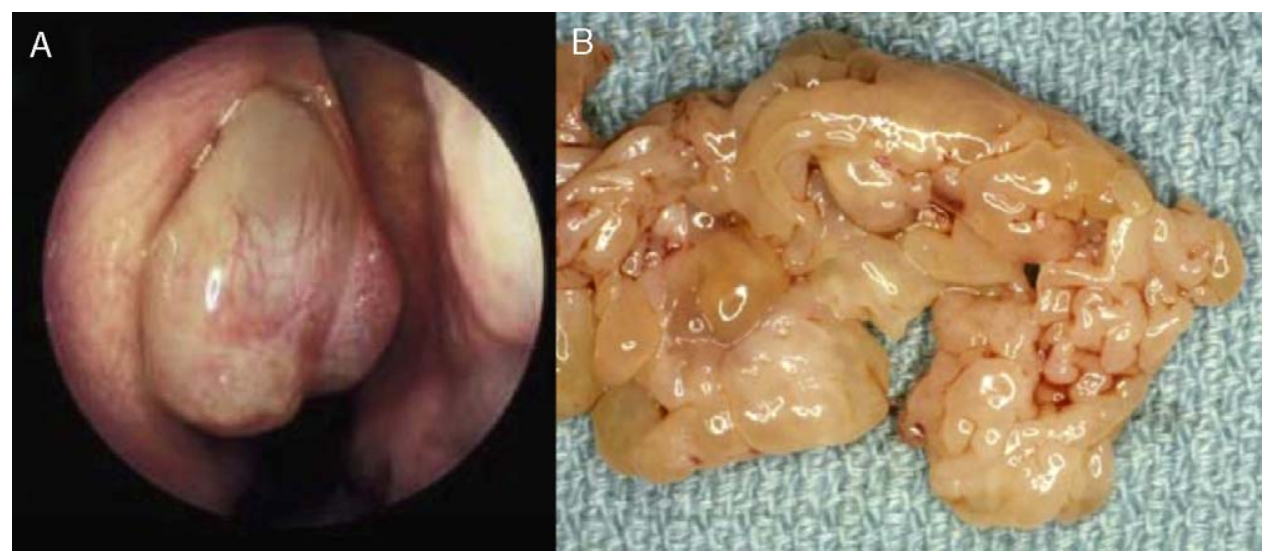

Figura 1 - Aspecto endoscópico (A) y macroscópico (B) del papiloma invertido. 

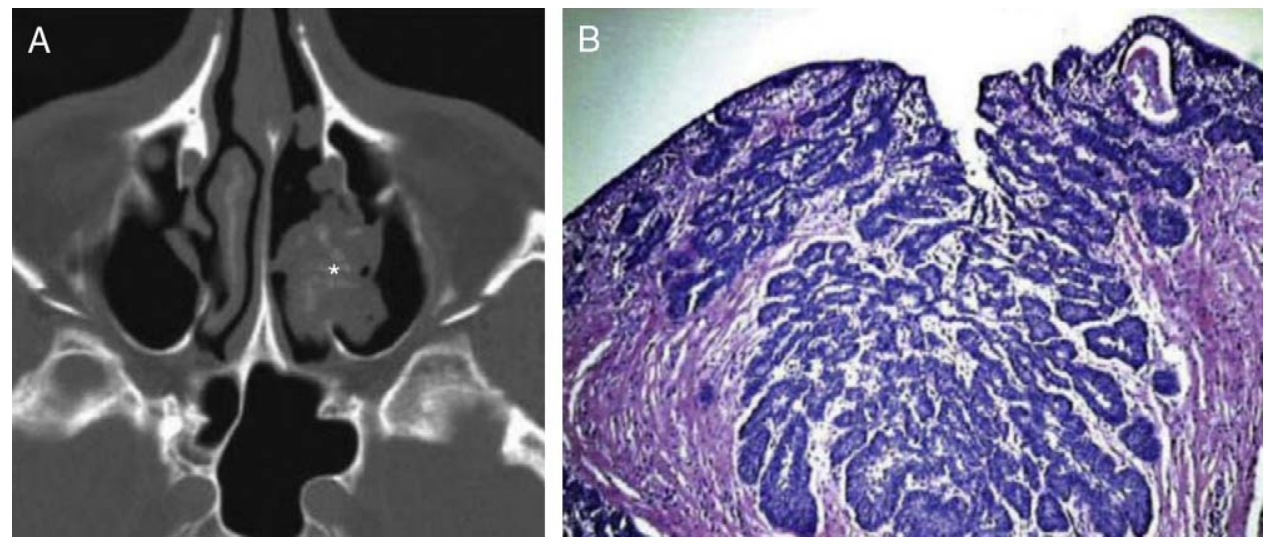

Figura 2 - TC de senos paranasales con papiloma invertido maxilar (A) y aspecto microscópico de papiloma invertido con densa hipercelularidad de la superficie epitelial e invaginaciones crípticas dentro del estroma subyacente (B).

combinado, y $2(13,4 \%)$ a cirugía endonasal. El tiempo medio de la recidiva fue de 14 meses.

Seis pacientes presentaron complicaciones, 5 (83,3\%) del grupo tratado endoscópicamente: 3 formaron sinequias, 1 mucocele y 1 con fístula de LCR; y uno $(16,7 \%)$ por abordaje externo presentó fistula oroantral. No hubo ninguna defunción debido a las complicaciones quirúrgicas.

La malignización ocurrió solo en 1 paciente $(6,6 \%)$ operado en primera intención por vía externa (etmoidectomía paranasal) y luego por vía endoscópica. Los pacientes de esta serie fueron seguidos al menos durante 36 meses.

\section{Discusión}

En nuestro estudio el sexo, edad media, síntomas y localización más frecuente del PI se correlaciona con la gran mayoría de la literatura revisada ${ }^{1-4,8-11}$. Históricamente fue propuesta la cirugía y radioterapia coadyuvante pero debido a las altas tasas de efectos adversos se desestimó esta última ${ }^{5}$, pudiendo afirmarse actualmente que el abordaje endoscópico exclusivo o combinado externamente ha probado ser eficiente al disminuir las tasas de recurrencia comparado con sólo el tradicional abordaje externo ${ }^{2,6,9,11}$. Así, la tendencia europea según recientes metaanálisis revela que los resultados con abordaje endoscópico son buenos, incluso mejores que por vía externa con respecto al control tumoral, ausencia de incisión facial, ingreso hospitalario y reducción del dolor postoperatorio ${ }^{6}$.

El manejo quirúrgico agresivo primario intentando remover por completo el tumor junto con amplios márgenes de mucosa saludable son las premisas de la terapia actual, sin embargo, recientemente se ha descrito la preservación quirúrgica del cornete inferior al realizar la maxilectomia medial, con la intención de ser más fisiológicamente conservador y evitar la sensación de congestión nasal postoperatoria originada por una posible rinitis seca y/o atrófica ${ }^{10}$.

La evaluación de la resecabilidad endoscopica antes de la cirugía es hecha por la combinación de exploración endoscópica (fig. 1 A) y técnicas de imagen (fig. 2 A). Nosotros realizamos TC en todos los pacientes y reservamos la RM para aquellos en los que era difícil distinguir la lesión de patología inflamatoria obstructiva.
No existen dudas sobre el elevado índice de recidivas, cuya mayoría se presenta dentro de los 2 primeros años y que se cree se deben generalmente a la resección incompleta ${ }^{9}$, considerando la experiencia del cirujano con el endoscopio como un gran condicionante de esta posibilidad ${ }^{10,11}$. Nuestra tasa de recidivas está probablemente en relación a la extensión, multifocalidad y al empleo en los primeros años de abordaje externo, ya que al resecarse sólo endoscópicamente se equipara con otras publicaciones ${ }^{1-3,10}$.Nuestros resultados en cuanto a recidivas se corresponden con los encontrados en la literatura nacional ${ }^{1,2,4,10}$ e internacional ${ }^{8,9,11,12}$; donde el tratamiento quirúrgico endoscópico es de elección sin descartar el abordaje externo cuando la extensión de la tumoración rebase los límites asequibles endoscópicamente. Por su tendencia acusada a recidivar, se recomienda un seguimiento anual endoscópico y, pruebas de imagen sólo si observamos hallazgos patológicos en la exploración endoscópica. El estudio de la pieza anatomopatológica es fundamental para confirmar el diagnóstico (fig. 2 B). En muchos de los centros con extendida experiencia la gran mayoría de los casos pueden ser manejados endoscópicamente, siendo en nuestro medio la opción actual más viable.

\section{Conflicto de intereses}

Los autores declaran no tener ningún conflicto de intereses.

\section{B I B L I O GR A F Í A}

1. Díaz Molina JP, Llorente Pendás JL, Rodrigo Tapia JP, Álvarez Marcos C, Obeso Agüera S, Suárez Nieto C. Papilomas invertidos rinosinusales, Revisión de 61 casos. Acta Otorrinolaringol Esp. 2009;60:402-8.

2. Llorente Pendás JL, Suárez Fente V, Suárez Nieto C. Papilomas invertidos nasosinusales. Acta Otorrinolaringol Esp. 2007;58:78-83.

3. Gras Cabrerizo JR, Montserrat Gili JR, Massegur Solench H, León Vintró X, De Juan J, Fabra Llopis JM. Management of sinonasal inverted papilomas and comparison of classification staging systems. Am J Rhinol Allergy. 2010;24:66-9. 
4. Henríquez M, Altuna X, Zulueta A, Gorostiaga F, Algaba J. Papiloma invertido: Tratamiento y evolución. Acta Otorrinolaringol Esp. 2003;54:242-8.

5. Sauter A. Focal malignancy in sinonasal inverted papiloma Is postoperative radiotherapy recomendable? Oral Oncology. 2011 [Epub ahead of print].

6. Carta F, Verillaud B, Herman P. Role of endoscopic approach in the management of inverted papilloma. Curr Opin Otolaryngol Head Neck Surg. 2011;19:21-4.

7. Huang CC, Lee TJ, Chang PH, Lee YS, Chuang CC, Jhang YJ, et al. Desmoglein 3 is overexpressed in inverted papilloma and squamous cell carcinoma of sinonasal cavity. Laryngoscope. 2010;120:26-9.

8. Mackle T, Chambon G, Garrel R, Meieff M, Crampette L. Endoscopic treatment of sinonasal papilloma: a 12 year review. Acta Oto-Laryngologica. 2008;128:670-4.
9. Lombardi D, Tomenzoli D, Buttà L, Bizzoni A, Farina D, Sberze F, et al. Limitations and complications of endoscopic surgery for treatment for sinonasal inverted papilloma: a reassessment after 212 cases. Head Neck. 2010 [Epub ahead of print].

10. Gras Cabrerizo JR, Massegur Solench H, Pujol Olmo A, Montserrat Gili JR, Ademá Alcover JM, Zarraonandia Andraca I. Endoscopic medial maxillectomy with preservation of inferior turbinate: how do we do it? Eur Arch Otorhinolaryngol. 2011;268:389-92.

11. Sham CL, Woo JK, van Hasselt CA, Tong MC. Treatment results of sinonasal inverted papilloma: an 18-year study. Am J Rhinol Allergy. 2009;23: 203-11.

12. Krouse JH. Development of a staging system for inverted papilloma. Laryngoscope. 2000;110:965-8. 